Article

\title{
Desiderata for Fractional Derivatives and Integrals
}

\author{
Rudolf Hilfer ${ }^{1}$ and Yuri Luchko ${ }^{2, *}$
}

1 ICP, Fakultät für Mathematik und Physik, Universität Stuttgart, Allmandring 3, 70569 Stuttgart, Germany; hilfer@icp.uni-stuttgart.de

2 Fachbereich Mathematik-Physik-Chemie, Beuth Hochschule für Technik Berlin, Luxemburger Str. 10, 13353 Berlin, Germany

* Correspondence: luchko@beuth-hochschule.de

Received: 11 January 2019; Accepted: 25 January 2019; Published: 4 February 2019

Abstract: The purpose of this brief article is to initiate discussions in this special issue by proposing desiderata for calling an operator a fractional derivative or a fractional integral. Our desiderata are neither axioms nor do they define fractional derivatives or integrals uniquely. Instead they intend to stimulate the field by providing guidelines based on a small number of time honoured and well established criteria.

Keywords: fractional derivatives; fractional integrals; fractional calculus

MSC: 26A33; 34A08; 34K37; 35R11; 44A40

A list of six desiderata ${ }^{1}$ is proposed that in our opinion would justify calling an operator $D^{\alpha}$ (or $I^{\alpha}$ ) a fractional derivative (or a fractional integral) of non-integer order $\alpha \notin \mathbb{N}$. Derivatives and integrals of fractional order have a long history and, up until the recent proliferation of novel fractional derivatives, most definitions and interpretations of fractional operators seem to implicitly assume the desiderata of an operational calculus as formulated in this article.

Mathematical terms used in the formulation of our desiderata are defined in Appendix A. A family $\left\{D^{\alpha}, I^{\alpha}\right\}$ of operators with $\alpha \in \mathbb{Q}, \mathbb{R}$ or $\mathbb{C}$ is proposed to be called a family of fractional derivatives $D^{\alpha}$ and integrals $I^{\alpha}$ of order $\alpha$ (with $\left.\operatorname{Re} \alpha \geq 0\right)^{2}$ if and only if it satisfies the following six desiderata:

(a) Integrals $I^{\alpha}$ and derivatives $D^{\alpha}$ of fractional order $\alpha$ should be linear operators on linear spaces ${ }^{3}$.

(b) On some subset ${ }^{4} \mathrm{G}_{(\mathrm{b})} \subset \mathrm{D}\left(I^{\alpha}\right) \cap I^{\beta}\left[\mathrm{D}\left(I^{\beta}\right)\right] \cap \mathrm{D}\left(I^{\alpha+\beta}\right) \neq \varnothing$ the index law (semigroup property)

$$
\left(I^{\alpha} \circ I^{\beta}\right) f=I^{\alpha+\beta} f
$$

holds true for $\operatorname{Re} \alpha \geq 0$ and $\operatorname{Re} \beta \geq 0$, where $\mathrm{D}\left(I^{\alpha}\right)$ denotes the domain of $I^{\alpha}$, and $\circ$ denotes composition of operators.

(c) Restricted to a suitable subset $\mathrm{G}_{(\mathrm{c})} \subset \mathrm{D}\left(I^{\alpha}\right)$ of the domain of $I^{\alpha}$ the fractional derivatives $D^{\alpha}$ of order $\alpha$ operate as left inverses

$$
D^{\alpha} \circ I^{\alpha}=1_{\mathrm{G}_{(\mathrm{c})}}
$$

for all $\alpha$ with $\operatorname{Re} \alpha \geq 0$, where $1_{\mathrm{G}_{(\mathrm{c})}}$ is the identity on $\mathrm{G}_{(\mathrm{c})}$.

1 properties to be desired.

2 It is common to use only one of the symbols $I$ or $D$ in the sense that either $D^{\alpha}=I^{-\alpha}$ or $I^{\alpha}=D^{-\alpha}$. In this paper we keep the distinction between $I$ and $D$ by assuming $\operatorname{Re} \alpha \geq 0$ unless otherwise specified. This entails discussing the case $\operatorname{Re} \alpha=0$ separately whenever necessary.

3 Dependencies of $I^{\alpha}$ and $D^{\alpha}$ on other parameters are usually present, but notationally suppressed.

4 Here the index (b) refers to desideratum (b). The same applies in desiderata (d)-(f) below. 
(d) There is a subset $\mathrm{G}_{(\mathrm{d})} \subset \mathrm{D}\left(D^{\alpha}\right)$ of the domain of $D^{\alpha}$ such that the limits

$$
\begin{array}{ll}
g^{1}=D^{1} f=\lim _{\alpha \rightarrow 1} D^{\alpha} f, & f \in \mathrm{G}_{(\mathrm{d}),} \\
g^{0}=D^{0} f=\lim _{\alpha \rightarrow 0} D^{\alpha} f, & f \in \mathrm{G}_{(\mathrm{d}),}
\end{array}
$$

exist in some sense and define linear maps $D^{1}: \mathrm{G}_{(\mathrm{d})} \rightarrow \mathrm{G}_{(\mathrm{d})}$ resp. $D^{0}: \mathrm{G}_{(\mathrm{d})} \rightarrow \mathrm{G}_{(\mathrm{d})}$.

(e) The limiting map $D^{0}=1_{\mathrm{G}_{(\mathrm{d})}}$ is the identity on $\mathrm{G}_{(\mathrm{d})}$, i.e., $g^{0}=f$;

(f) The limiting map $D^{1}=D$ is a derivation on $\mathrm{G}_{(\mathrm{d})}$. This means it is possible to define a multiplication $\cdot: G_{(d)} \times G_{(d)} \rightarrow G_{(d)}$ on $G_{(d)}$ such that the Leibniz rule

$$
D(f \cdot g)=g \cdot(D f)+f \cdot(D g)
$$

holds for all $f, g \in \mathrm{G}_{(\mathrm{d})}$.

If the semigroup law (1) can be extended to all $\alpha \in \mathbb{R}$ or $\alpha \in \mathbb{C}$, we propose to speak of fractional calculus. Our desiderata are obviously inspired by operational calculus. Recall that an operational calculus is a continuous one-to-one mapping between an algebra of functions and an algebra of operators such that the neutral elements match and algebraic relations are preserved. Extending the algebra from polynomial functions to convergent power series suffices for an operational calculus. More singular functions, namely non-analytic power functions, are required for fractional calculus.

Desiderata differing substantially from those above have been formulated in [1] (p. 5) and [2] (p. 5). Envisaging exclusively analytic functions the criteria given in [1] (p. 5) are extremely restrictive. In theory and applications it is nowadays imperative to include more general functions, measures and also distributions into the purview.

Given the extreme restrictions in [1] (p. 5) a more recent proposal [2] went to opposite extremes. Little or no attention is given to a domain of definition for the fractional derivatives in [2]. Our desiderata for fractional derivatives again differ substantially from those in [2]. Rather than requiring some form of the generalized Leibniz rule ${ }^{2} \mathrm{P} 5$ in [2] for all $\alpha$ we desire the Leibniz rule only for $\alpha=1$, and that differs not only from ${ }^{2} \mathrm{P} 5$, but also from ${ }^{2} \mathrm{P} 3$. In addition the identity rule ${ }^{2} \mathrm{P} 2$ in [2] does not restrict the admissible operators at all. As long as there is no continuity in $\alpha$ or a well defined limit, the identity rule can always be fulfilled, simply by setting $D^{0}=1$. More generally, Ref. [2] seems to neglect parameters other than $\alpha$, or the topological and operator-theoretic implications of the limit in Equation (2) [2].

Our desiderata do not include non-locality of fractional derivatives. Fractional derivatives, that are local operators, were introduced in [3,4] and are discussed further in [5] and Section 7 of [6]. Contrary to ${ }^{2} \mathrm{P} 3$ in [2] we do not constrain the limits $\alpha \rightarrow n$ with $n \in \mathbb{N}$ for $n \geq 2$, because we wish to allow more generality.

To illustrate our desiderata in a simple case consider fractional operators of Riemann-Liouville type for complex valued functions $f:[a, b] \rightarrow \mathbb{C}$ on a compact interval $[a, b] \subset \mathbb{R}$ with $-\infty<a<b<\infty$ when the fractional order $\alpha$ is real and restricted to $0 \leq \alpha \leq 1$. The (right-sided) Riemann-Liouville fractional integrals $I_{a+}^{\alpha}$ of order $\alpha \geq 0$ with lower limit $a$ are defined by setting $I_{a+}^{0} f:=f$ for $\alpha=0$ and

$$
\left(I_{a+}^{\alpha} f\right)(x):=\frac{1}{\Gamma(\alpha)} \int_{a}^{x}(x-y)^{\alpha-1} f(y) \mathrm{d} y
$$

for $\alpha>0$ and $x \leq b$. The (right-sided) Riemann-Liouville-type fractional derivatives $D_{a+}^{\alpha}$ of order $0 \leq \alpha \leq 1$ are defined as [5] (p. 434)

$$
\left(D_{a+}^{\alpha, \beta} f\right)(x)=\left(I_{a+}^{\beta(1-\alpha)} \frac{\mathrm{d}}{\mathrm{d} x} I_{a+}^{(1-\beta)(1-\alpha)} f\right)(x)
$$


where the number $0 \leq \beta \leq 1$ parametrizes different types of fractional derivatives. The classical Riemann-Liouville derivative is of type $\beta=0$, while the popular Liouville-Caputo derivative has type $\beta=1[7]$ (p. 10).

Both operator families are linear and desideratum (a) can be fulfilled on numerous linear spaces due to the compactness of $[a, b]$. Examples are the Lebesgue spaces $\mathrm{L}^{p}([a, b])$ with $1 \leq p \leq \infty$ or Hölder spaces $C^{\gamma}([a, b])$ with $\gamma>0$. Desideratum (c) holds e.g. for $\mathrm{G}_{(\mathrm{c})}=\mathrm{C}^{\gamma}([a, b])$ with $\gamma>\alpha+\beta-\alpha \beta$ and $\alpha+\beta-\alpha \beta \neq 1$. The Riemann-Liouville fractional integrals (extended from $0 \leq \alpha \leq 1$ to $\alpha \geq 0$ ) are a strongly continuous semigroup of operators with respect to the parameter $\alpha \geq 0$, and obey the index law (1) in desideratum (b) for all $\alpha, \beta \geq 0$ on $\mathrm{G}_{(\mathrm{b})}=\mathrm{L}^{p}([a, b])$ or suitable subspaces. The desiderata (d), (e) and (f) can then be derived with the help of the semigroup property. For the Riemann-Liouville operators they hold e.g. for smooth (infinitely often differentiable) functions $f \in \mathrm{G}_{(\mathrm{d})}=\mathrm{C}^{\infty}([a, b])$.

For infinite intervals or for generalized functions the problem of domains may become more involved and our desiderata may become more restrictive. As an example consider the family of symmetric Riesz operators

$$
\left(I^{\alpha} f\right)(x):=\frac{1}{2 \Gamma(\alpha) \cos (\alpha \pi / 2)} \int_{-\infty}^{\infty}|x-y|^{\alpha-1} f(y) \mathrm{d} y
$$

on the real line. In this case the limiting operator $D^{1}=\lim _{\alpha \rightarrow-1} I^{\alpha}$ is again well defined, but does not fulfill desideratum (f). Instead, it fulfills Leibniz' formula for $D^{n}(f g)$ with $n=2$, i.e.,

$$
D^{1}\left[D^{1}(f g)\right]=g D^{1}\left(D^{1} f\right)+2\left(D^{1} f\right)\left(D^{1} g\right)+f D^{1}\left(D^{1} g\right) .
$$

We propose to call such operators obeying Leibniz formula for $D^{n}(f g)$ with $n \geq 2$ pseudofractional derivatives or fractional pseudoderivatives.

Of course, our desiderata do not define fractional derivatives and integrals in a unique way. Still, they considerably restrict the set of admissible operators as seen above. In our opinion the above desiderata formulate crucial constraints for the development of a meaningful mathematical theory of fractional calculus and its reasonable applications.

Much work has been done on mathematical interpretations of fractional derivatives and integrals. The results are documented in numerous texts and treatises (see [6,8] for recent reviews). It seems however, that the connection (or not) of classical and recent fractional calculi with historical and contemporary forms of operational and functional calculi such as Heaviside-Mikusinski calculus, Dunford-Schwarz calculus, or Hille-Phillips calculus is a rich source of numerous open problems whose speedy solution would seem pertinent to advance and ultimately consolidate the field. We hope that the desiderata above are sufficiently restrictive to initiate a discussion of these pressing problems, and thereby stimulate readers and contributors to address some of these open problems in their areas of expertise and interest.

\section{Appendix A}

For the convenience of readers from non-mathematical disciplines we recall some definitions: A real (or complex) linear space (or vector space) over the field $\mathbb{R}$ (or $\mathbb{C}$ ) of real (or complex) numbers is a non-empty set $\mathrm{X}$ with two operations called addition and scalar multiplication fulfilling the usual rules of vector addition and multiplication of vectors with numbers ${ }^{5}$.

5 (a) for all $f, g \in \mathbf{X}$ also $f+g \in \mathbf{X}$, (b) $f+g=g+f$, (c) $f+(g+h)=(f+g)+g$, (d) there exists an element $0 \in \mathbf{X}$ (called origin ) such that $f+0=f$ for all $f \in \mathbf{X}$, (e) for all $f \in \mathbf{X}$ there is an element $-f \in \mathbf{X}$ such that $f+(-f)=0$ (f) for all $a \in \mathbb{R}$ (or $a \in \mathbb{C}$ ) and $f \in \mathbf{X}$ an element $a f \in \mathbf{X}$ is defined, (g) for all $a \in \mathbb{R}$ (or $a \in \mathbb{C}$ ) and $f, g \in \mathbf{X}$ one has $a(f+g)=a f+a g$, (h) for all $a, b \in \mathbb{R}$ (or $a, b \in \mathbb{C}$ ) and $f \in \mathbf{X}$ one has $(a+b) f=a f+b f$, (i) for all $a, b \in \mathbb{R}$ (or $a, b \in \mathbb{C}$ ) and $f \in \mathbf{X}$ one has $a(b f)=(a b) f$, and (j) $1 f=f$ for all $f \in \mathbf{X}$. 
Let $\mathrm{X}, \mathrm{Y}, \mathrm{Z}$ be linear spaces (vector spaces). A linear operator $A: \mathrm{X} \rightarrow \mathrm{Y}$ is a linear subspace of the direct sum $X \oplus Y$, where

$$
\mathrm{X} \oplus \mathrm{Y}:=\{(f, g): f \in \mathrm{X}, g \in \mathrm{Y}\}
$$

is the linear space of pairs $(f, g)$ with $f \in \mathrm{X}, g \in \mathrm{Y}$ and addition defined as $\left(f_{1}, g_{1}\right)+\left(f_{2}, g_{2}\right)=$ $\left(f_{1}+f_{2}, g_{1}+g_{2}\right)$ for all $f_{i} \in \mathbf{X}$ and $g_{i} \in \mathrm{Y}$. The identity operator $1_{\mathbf{X}}: \mathrm{X} \rightarrow \mathrm{X}$ is defined as

$$
1_{\mathrm{X}}=1:=\{(f, f): f \in \mathrm{X}\} .
$$

The domain $\mathrm{D}(A)$ and range $\mathrm{R}(A)$ of a linear operator $A: \mathrm{X} \rightarrow \mathrm{Y}$ are

$$
\begin{aligned}
& \mathrm{D}(A):=\{f \in \mathrm{X}: \exists g \in \mathrm{Y} \text { s.t. }(f, g) \in A\} \\
& \mathrm{R}(A):=\{g \in \mathrm{Y}: \exists f \in \mathrm{X} \text { s.t. }(f, g) \in A\} .
\end{aligned}
$$

The inverse $A^{-1}$ of $A$ is defined as

$$
A^{-1}:=\{(g, f) \in \mathrm{Y} \oplus \mathbf{X}:(f, g) \in A\}
$$

with domain $\mathrm{D}\left(A^{-1}\right)=\mathrm{R}(A)$. For $A, B \in \mathrm{X} \oplus \mathrm{Y}$ their sum is defined as

$$
A+B:=\{(f, g+h) \in \mathbf{X} \oplus \mathbf{Y}:(f, g) \in A,(g, h) \in B\}
$$

with $\mathrm{D}(A+B)=\mathrm{D}(A) \cap \mathrm{D}(B)$. For $A \in \mathrm{X} \oplus \mathrm{Y}, B \in \mathrm{Y} \oplus \mathrm{Z}$ the composition $B \circ A: \mathrm{X} \rightarrow \mathrm{Z}$ is the linear operator defined as

$$
B \circ A:=\{(f, h) \in \mathbf{X} \oplus \mathbf{Z}: \exists g \in \mathbf{Y} \text { s.t. }(f, g) \in A \text { and }(g, h) \in B\}
$$

with $\mathrm{D}(B \circ A)=\{f \in \mathrm{D}(A): \exists g \in \mathrm{D}(B)$ s.t. $(f, g) \in A\}$.

Let $1 \leq p<\infty$ be a fixed real number. The Lebesgue space $\mathrm{L}^{p}([a, b])$ consists of those Lebesgue measurable functions $f:[a, b] \rightarrow \mathbb{C}$ on the intervall $[a, b]=[a, b] \subset \mathbb{R}$ for which the norm

$$
\|f\|_{p}=\left(\int_{[a, b]}|f(x)|^{p} \mathrm{~d} x\right)^{1 / p}
$$

is finite. For $p=\infty$ the space $\mathrm{L}^{\infty}([a, b])$ is the set of all Lebesgue measurable functions such that


zero (called essential supremum). The space $\mathrm{B}([a, b])$ consists of all bounded functions on $[a, b]$. Its norm is $\|f\|_{\mathrm{B}}=\sup _{x \in[a, b]}|f(x)|$. The space $\mathrm{C}([a, b])=\mathrm{C}^{0}([a, b])$ consists of all continuous functions. Its norm is again $\|f\|_{\mathrm{B}}=\sup _{x \in[a, b]}|f(x)|$ because continuous functions on a compact interval are also bounded. For $0<\gamma \leq 1$ and $f:[a, b] \rightarrow \mathbb{C}$ the number

$$
\operatorname{Höl}_{\gamma}(f,[a, b]):=\sup \left\{\frac{|f(x)-f(y)|}{|x-y|^{\gamma}} ; x, y \in[a, b], x \neq y\right\} \in[0, \infty]
$$

is called Hölder constant of $f$ on $[a, b]$ of Hölder order $\gamma$. The Hölder space $\mathrm{C}^{\gamma}([a, b])$ is defined as

$$
\mathrm{C}^{\gamma}([a, b]):=\left\{f \in \mathrm{C}([a, b]): \operatorname{Höl}_{\gamma}(f,[a, b])<\infty\right\}
$$


and is a Banach space for the norm

$$
\|f\|_{\mathrm{C}^{\gamma}}=\sup _{s \in[a, b]}|f(x)|+\operatorname{Höl}_{\gamma}(f,[a, b]) .
$$

For $\alpha=k+\gamma>1$ with $k=0,1,2, \ldots$ and $0<\gamma \leq 1$ it is defined as

$$
C^{\alpha}([a, b]):=\left\{f \in C^{k}([a, b]): \operatorname{Höl}_{\gamma}\left(f^{(k)},[a, b]\right)<\infty\right\}
$$

with

$$
\|f\|_{C^{\alpha}}=\|f\|_{C^{k}}+\left\|f^{(k)}\right\|_{C^{\gamma}}
$$

where $f^{(k)}$ is the $k$-th derivative of $f$.

A family $\{T(t)\}, t \geq 0$ of bounded linear operators $T(t): \mathrm{X} \rightarrow \mathrm{X}$ on a Banach space $\mathrm{X}$ is called a strongly continuous one-parameter semigroup if it satisfies:

(a) $T(0)=1_{\mathbf{X}}$

(b) $T(t) T(s)=T(t+s)$ for all $t, s \geq 0$.

(c) For every $x \in \mathrm{X}$ the orbit maps $y_{x}: t \mapsto y_{x}(t):=T(t) x$ are continuous from $[0, \infty)$ into $\mathrm{X}$.

\section{References}

1. Ross, B. A brief history and exposition of the fundamental theory of fractional calculus. In Fractional Calculus and its Applications; Ross, B., Ed.; Springer Verlag: Berlin, Germany, 1975; Volume 457, pp. 1-37.

2. Ortigueira, M.; Tenreiro-Machado, J. What is a fractional derivative? J. Comput. Phys. 2015, 293, 4-13. [CrossRef]

3. Hilfer, R. Thermodynamic Scaling Derived via Analytic Continuation from the Classification of Ehrenfest. Phys. Scr. 1991, 44, 321. [CrossRef]

4. Hilfer, R. Multiscaling and the Classification of Continuous Phase Transitions. Phys. Rev. Lett. 1992, 68, 190. [CrossRef] [PubMed]

5. Hilfer, R. Fractional Calculus and Regular Variation in Thermodynamics. In Applications of Fractional Calculus in Physics; Hilfer, R., Ed.; World Scientific: Singapore, 2000; p. 429.

6. Hilfer, R. Mathematical and physical interpretations of fractional derivatives and integrals. In Handbook of Fractional Calculus and Applications, Volume 1: Basic Theory; Kochubei, A., Luchko, Y., Eds.; De Gruyter: Berlin, Germany, 2019; p. 47.

7. Liouville, J. Mémoire sur quelques Questions de Geometrie et de Mecanique, et sur un nouveau genre de Calcul pour resoudre ces Questions. J. l'Ecole Polytech. 1832, XIII, 1.

8. Kochubei, A.; Luchko, Y. Basic FC operators and their properties. In Handbook of Fractional Calculus and Applications, Volume 1: Basic Theory; Kochubei, A., Luchko, Y., Eds.; De Gruyter: Berlin, Germany, 2019; p. 23. 